\title{
BMJ Open Analgesic efficacy and safety of ketamine after total knee or hip arthroplasty: a meta-analysis of randomised placebo-controlled studies
}

\author{
Bei Xu, ${ }^{1}$ Yilun Wang, ${ }^{\circ}$ Chao Zeng, ${ }^{1}$ Jie Wei, ${ }^{2,3}$ Jiatian $\mathrm{Li},{ }^{1}$ Ziying Wu, ${ }^{1}$ Hongyi He, ${ }^{1}$ \\ Guanghua Lei, ${ }^{1}$ Dongxing Xie, ${ }^{1}$ Xiang Ding ${ }^{1}$
}

To cite: Xu B, Wang Y, Zeng C, et al. Analgesic efficacy and safety of ketamine after total knee or hip arthroplasty: a metaanalysis of randomised placebocontrolled studies. BMJ Open 2019;9:e028337. doi:10.1136/ bmjopen-2018-028337

- Prepublication history and additional material for this paper are available online. To view please visit the journal (http:// dx.doi.org/10.1136/bmjopen2018-028337).

$\mathrm{BX}$ and $\mathrm{YW}$ contributed equally. $\mathrm{DX}$ and $\mathrm{XD}$ contributed equally.

Received 03 December 2018

Revised 29 July 2019

Accepted 23 August 2019

Check for updates

(c) Author(s) (or their employer(s)) 2019. Re-use permitted under CC BY-NC. No commercial re-use. See rights and permissions. Published by BMJ.

${ }^{1}$ Orthopaedics, Xiangya Hospital, Central South University,

Changsha, China

${ }^{2}$ Health Management Center, Xiangya Hospital, Central South University, Changsha, China ${ }^{3}$ Department of Epidemiology and Health Statistics, Xiangya School of Public Health, Central South University, Changsha,

China

Correspondence to Dr Dongxing Xie; xdx1024@csu.edu.cn

Dr Xiang Ding; dingxiang@csu.edu.cn

\section{ABSTRACT}

Objective To examine the analgesic efficacy and safety of ketamine after total knee or hip arthroplasty.

Design Systematic review and meta-analysis.

Data sources PubMed, EMBASE and Cochrane Library from inception to 22 May 2019.

Eligibility criteria for selecting studies Randomised controlled trials comparing the efficacy and safety of ketamine with placebo for postoperative pain relief in patients undergoing total knee or hip arthroplasty.

Data extraction and synthesis Data (ie, pain intensity, morphine consumption, gastrointestinal and psychotic adverse effects) were extracted by two reviewers independently. The Cochrane Collaboration's recommended tool was used to determine the methodological quality of included studies.

Results A total of 10 studies were included. One of them was rated as low quality. Compared with placebo, intravenous ketamine was effective for pain relief during 0-8-hour (weighted mean difference (WMD) -1.21, $95 \% \mathrm{Cl}-1.45$ to $-0.98, \mathrm{p}<0.001$; three studies, 149 participants) but not during 8-24-hour postoperative periods, and effective for reduction of cumulative morphine consumption during both $0-24$-hour (WMD $-17.76,95 \% \mathrm{Cl}$ -31.25 to $-4.27, p=0.01$; five studies, 366 participants) and 0-48-hour (WMD $-21.79,95 \% \mathrm{Cl}-25.46$ to -18.11 , $\mathrm{p}<0.001$; four studies, 252 participants) postoperative periods, without increasing risks of gastrointestinal or psychotic adverse effects. The limited data available for intra-articular (WMD $-0.49,95 \% \mathrm{Cl}-0.70$ to -0.29 , $\mathrm{p}<0.001$; two studies, 83 participants) and epidural (WMD $-2.10,95 \% \mathrm{Cl}-3.30$ to $-0.90, \mathrm{p}<0.001$; one study, 20 participants) ketamine pointed to a significant reduction in pain intensity during 8-24-hour postoperative period. Conclusions Intravenous administration of ketamine is effective and safe for postoperative pain relief in patients undergoing total knee or hip arthroplasty. Nevertheless, the analgesic efficacy and safety of ketamine in such patients seem to vary by different administration routes and still warrant further studies to explore.

\section{INTRODUCTION}

The performance of total joint arthroplasty, a definitive treatment for patients with end-stage osteoarthritis, has increased
Strengths and limitations of this study

- This is the first systematic review and meta-analysis that directly investigates the efficacy and safety of ketamine in all feasible administration routes (ie, intravenous, intra-articular and epidural routes) after total knee or hip arthroplasty.

- All included studies adopted the randomised placebo-controlled design.

- Substantial heterogeneity was observed in some of the results. Although several studies had been eliminated from sensitivity analyses based on appropriate exclusion criteria, the source of heterogeneity was not identified.

- The meta-analysis technique was not fully applicable for investigating the efficacy and safety of intra-articular and epidural administration of ketamine due to the limited number of included studies.

dramatically over the past two decades and is expected to increase continuously with the ageing process of the population. ${ }^{1}$ Unfortunately, patients may experience severe postoperative pain, which can impede their rehabilitation and prolong the length of hospitalisation. ${ }^{1-3}$ As opioids and non-steroidal anti-inflammatory drugs are both commonly used, their adverse effects are frequently seen, especially in patients with chronic pain, who are often dissatisfied with regular analgesia. ${ }^{4}$ Patients with chronic pain tend to have a lower endurance against pain, and therefore are dependent on and tolerant to opioids. ${ }^{5}$ They typically demand a higher dose of analgesics, which may further reduce the level of safety and worsen the overall perioperative experience. ${ }^{4}$

Ketamine is a non-competitive N-methyl-D-aspartate (NMDA) receptor antagonist that has been widely used as an anaesthetic drug for the management of postoperative pain. $^{6-8}$ Several reviews and meta-analyses have been carried out focusing on its benefit 
and risk profiles. ${ }^{9}{ }^{10}$ However, with different administration routes of ketamine and types of surgery, the overall findings appear contradictory. More recently, a Cochrane review conducted by Brinck and colleagues showed that the perioperative intravenous ketamine might reduce postoperative analgesic consumption and pain intensity; this finding has a significant meaning in guiding clinical practice. ${ }^{9}$ Nevertheless, to our best knowledge, few studies have investigated the beneficial effect of ketamine on patients after total knee or hip arthroplasty, who are likely to experience severe postoperative pain and suffer from impeded rehabilitation and prolonged hospitalisation. ${ }^{1-3}$ While most researchers reported that intravenous ketamine could reduce postoperative pain intensity and analgesic consumption after total joint arthroplasty, ${ }^{11-14}$ there were still several studies disagreeing with such efficacy. ${ }^{1516}$ Moreover, as a specific administration route after joint surgery, the feasibility of implementing intra-articular administration of ketamine also remains to be a subject of debate. ${ }^{17} 18$

To facilitate clinical decision making, healthcare providers need to understand how well ketamine works as an analgesic and what the incidence of its adverse effects is. With newly accumulated evidence, the purpose of this meta-analysis was to evaluate the therapeutic benefits of ketamine in different administration routes for pain control after total knee or hip arthroplasty, as well as to elucidate its adverse effects. It was hypothesised that ketamine acted overall as an effective analgesic after total knee or hip arthroplasty without increasing the incidence of adverse effects.

\section{MATERIALS AND METHODS}

\section{Search strategy}

This meta-analysis was performed in accordance with the Preferred Reporting Items for Systematic Reviews and Meta-analyses statement. ${ }^{19}$ PubMed, EMBASE and Cochrane Library were searched from inception to 22 May 2019 for relevant studies comparing ketamine with placebo in patients after total knee or hip arthroplasty surgery. Search terms were 'arthroplasty', 'ketamine' and 'randomised placebo-controlled trials'. The full electronic search strategy was available in online supplementary appendix 1 . No restriction was imposed. The corresponding author of the potential relevant study was contacted if the full-text was not available.

\section{Study selection}

The citations and abstracts generated by the literature search were reviewed by two researchers independently. Inclusion criteria were as follows: (1) patients undergoing total knee or hip arthroplasty surgery; (2) administration of ketamine for postoperative pain relief; (3) administration of saline in the control group and (4) randomised controlled trials. Exclusion criteria were as follows: (1) vitro studies, animal studies, reviews, letters and case reports; (2) experimental or control group received additional treatments; (3) data not available for extraction and (4) unavailability of the full text. A consensus procedure was conducted for study selection. If consensus was not reached, a third reviewer would make a judgement.

\section{Data extraction}

The outcomes and characteristics of each included study were screened independently by two researchers. The mean and SD of outcomes, if not reported, was estimated based on sample size, median and the range. ${ }^{20}$ Data were also extracted from figures by using GetData V.2.20. The following background information was extracted from the included studies: first author's name, year of publication, mean age, gender ratio, sample size, doses of ketamine, follow-up time point, type of injection, start time of injection and type of arthroplasty. If there were more than two groups in one study, only data from the ketamine and placebo group were extracted. Pain intensity was chosen as the primary outcome in this meta-analysis. Secondary outcome measures of the present study were cumulative morphine consumption and adverse effects.

\section{Risk of bias assessment}

The Cochrane Collaboration's recommended tool was used to assess the methodological quality of included studies. ${ }^{21}$ Seven potential risks of bias were judged in the assessment tool: random sequence generation, allocation concealment, blinding of participants, blinding of outcome assessment, incomplete outcome data, selective reporting and other bias (mainly included the conflict of interests). Studies with three or more high risks of bias were considered as poor methodological quality. ${ }^{22}$ Two researchers conducted risk of bias assessment independently. Disagreements were resolved through discussions.

\section{Statistical analysis}

Quantitative analysis was undertaken for pain intensity reported as a visual analogue scale (VAS) score and cumulative morphine consumption. We calculated weighted mean difference (WMD) and their corresponding 95\% CIs. Postoperative pain intensity was analysed in the 0-8-hour, and 8-24-hour postoperative periods. To facilitate and standardise pooling of data, VAS scores at the last follow-up time point during the early (ie, $0-8$-hour) and late (ie, 8-24-hour) postoperative periods were extracted for analysis. All VAS scores were converted to a scale ranging from 0 to 10 . Cumulative morphine consumption was analysed in 0-24-hour and 0-48-hour postoperative periods. Dichotomous data on the adverse effects were summarised using the risk ratio (RR) and their corresponding $95 \%$ CIs. Adverse effects were classified into two groups: psychotic and gastrointestinal adverse effects.

The homogeneity of the effect size across trials was tested by the $\mathrm{Q}$ statistic $(\mathrm{p}<0.05$ was considered heterogeneous). If there was significant heterogeneity among the studies, the random-effects model was used; otherwise, the fixed-effects model was considered acceptable. 


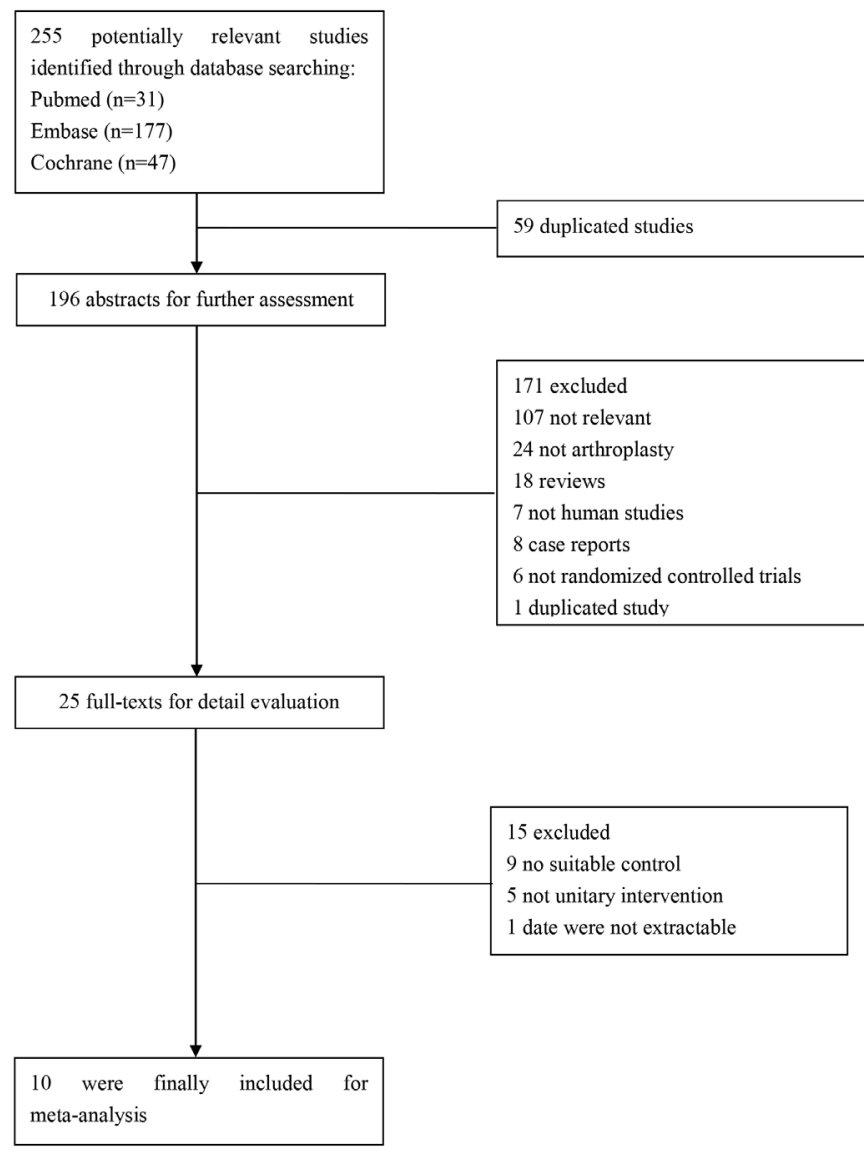

Figure 1 Flow diagram of screened, excluded and analysed articles.

We also examined the $\mathrm{I}^{2}$ statistic, which measures the proportion of the total variation across studies as a result of heterogeneity expressed as a percentage $\left(\mathrm{I}^{2}>50 \%\right.$ was considered to be heterogeneous).

Begg's tests ${ }^{23}$ and funnel plots were performed to assess publication bias. Statistical analyses were performed using Review Manager V.5.2 software (RevMan V.5.2, The Cochrane Collaboration, Oxford, UK) and STATA V.11.0 (StataCorp LP). A p value $<0.05$ was considered to be statistically significant unless otherwise specified.

\section{Patient and public involvement}

No patients or members of public were involved in the present study. No patients were asked to advise on the interpretation or writing up of results. The results of the present research will be communicated to the relevant patient community.

\section{RESULTS}

A total of 255 articles were retrieved from the initial search. After in-depth review of 25 papers, 15 were excluded for a variety of predetermined reasons, such as lack of suitable control group, ketamine co-administered with other analgesics, or unavailability of data extraction. Eventually, 10 papers ${ }^{11-182425}$ were included in final analyses (figure 1). The characteristics of 10 included studies are presented in table 1 . Among these studies, $\operatorname{seven}^{11-1625}$ involve intravenous administration of ketamine and three involve either intra-articular or epidural administration of ketamine. ${ }^{17} 1824$ The methodological quality of the involved trials is shown in figures 2 and 3. It can be seen that most of them were of high quality according to Cochrane Collaboration's recommended tool, and only one study ${ }^{25}$ was rated as low quality. Seven studies adopted appropriate methods to generate the random sequence, and six studies claimed that the allocation concealment was achieved by sealed envelopes. Eight studies implemented blinding over both the participants and research personnel, and seven study implemented blinding over the assessors. Eight studies reported the percentage of participants withdrawn less than $10 \%$. Seven studies predefined outcomes reported adequately. High risk of bias due to other bias (eg, different surgeons and different rehabilitation procedures) was detected in seven studies.

\section{Pain intensity}

Nine studies ${ }^{11-18} 24$ were eligible for the assessment of acute postoperative pain intensity during early and late postoperative periods (figures 4 and 5 ). The pooled data showed that the intravenous administration of ketamine had significantly lower pain scores compared with the placebo during the early (WMD $-1.21,95 \%$ CI -1.45 to $-0.98, \mathrm{p}<0.001$; three studies, 149 participants), but not the late (WMD $-0.48,95 \%$ CI -1.13 to $0.17, \mathrm{p}=0.14$; six studies, 466 participants) postoperative period. Substantial heterogeneity was observed during the late $\left(\mathrm{I}^{2}=90 \%\right.$; $\mathrm{p}<0.001)$, but not the early $\left(\mathrm{I}^{2}=50 \%, \mathrm{p}=0.14\right)$ postoperative period. Sensitivity analysis was conducted to explore the potential sources of heterogeneity between the intravenous administration of ketamine group and the control group during the late postoperative period. With exclusion of any single study, the substantial heterogeneity did not change materially. Funnel plots did not reveal any substantial asymmetry (figure 6), and Begg's rank correlation test did not indicate publication bias among the included studies (early: $\mathrm{p}=0.296$; late: $\mathrm{p}=1.000$ ).

The pooled results of two included studies ${ }^{17} 18$ showed that the pain intensity in the intra-articular administration of ketamine group was significantly lower than that in the control group during the late postoperative period (WMD $-0.49,95 \%$ CI -0.70 to -0.29 , $\mathrm{p}<0.001$; two studies, 83 participants), with no substantial heterogeneity $\left(I^{2}=0 \%, p=0.63\right)$. However, no significant difference was observed between the two groups in terms of pain intensity during the early postoperative period (WMD $-0.12,95 \%$ CI -0.51 to $0.26, p=0.52$; two studies, 83 participants), and no substantial heterogeneity was observed $\left(I^{2}=0 \%, p=0.54\right)$. Funnel plots did not reveal any substantial asymmetry (figure 6), and Begg's rank correlation test did not indicate publication bias among the included studies (early: $\mathrm{p}=1.000$; late: $\mathrm{p}=1.000$ ).

Only one study, ${ }^{24}$ conducted on a small-sized sample, reported that the epidural administration of ketamine 


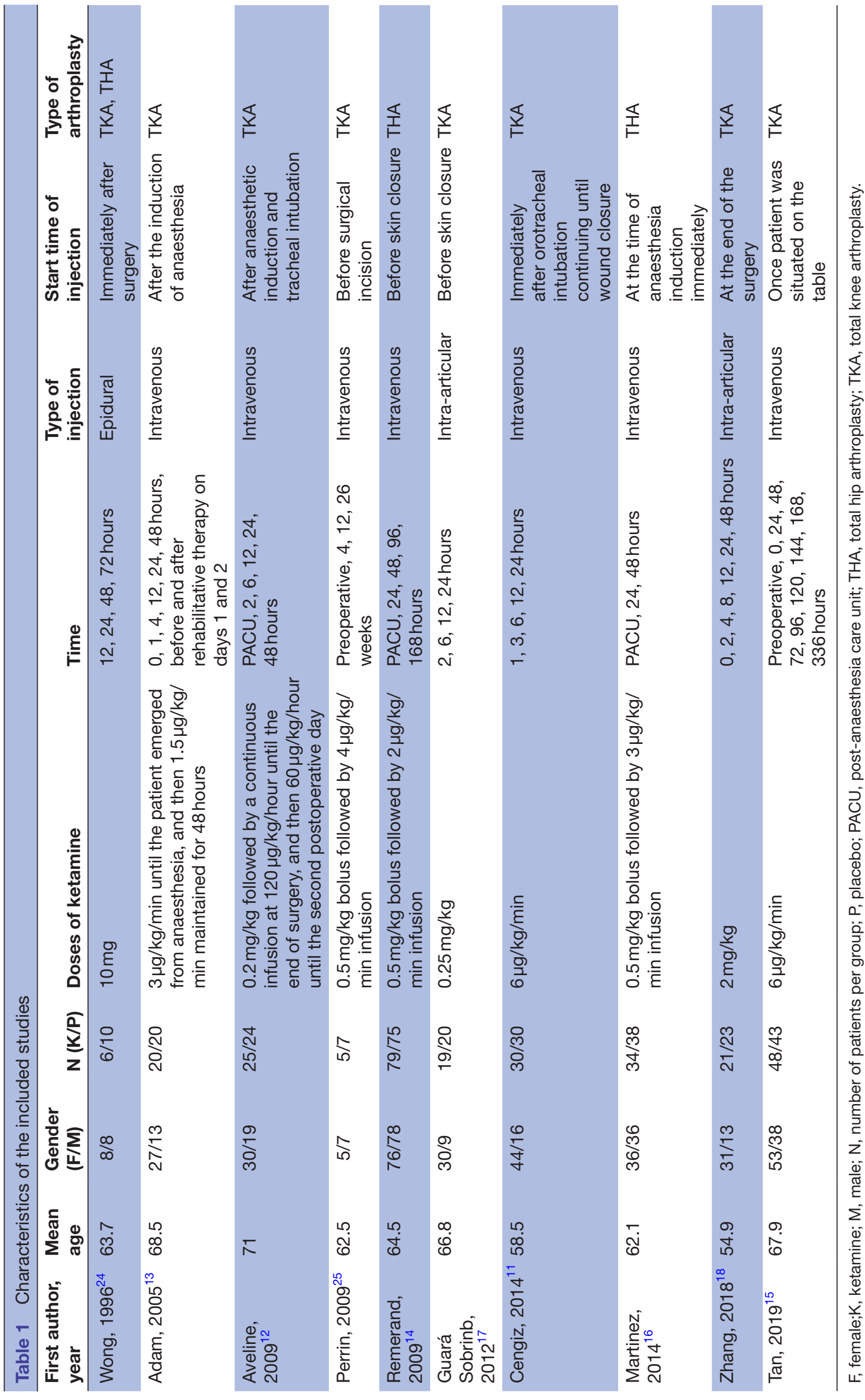

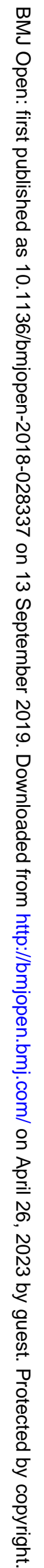




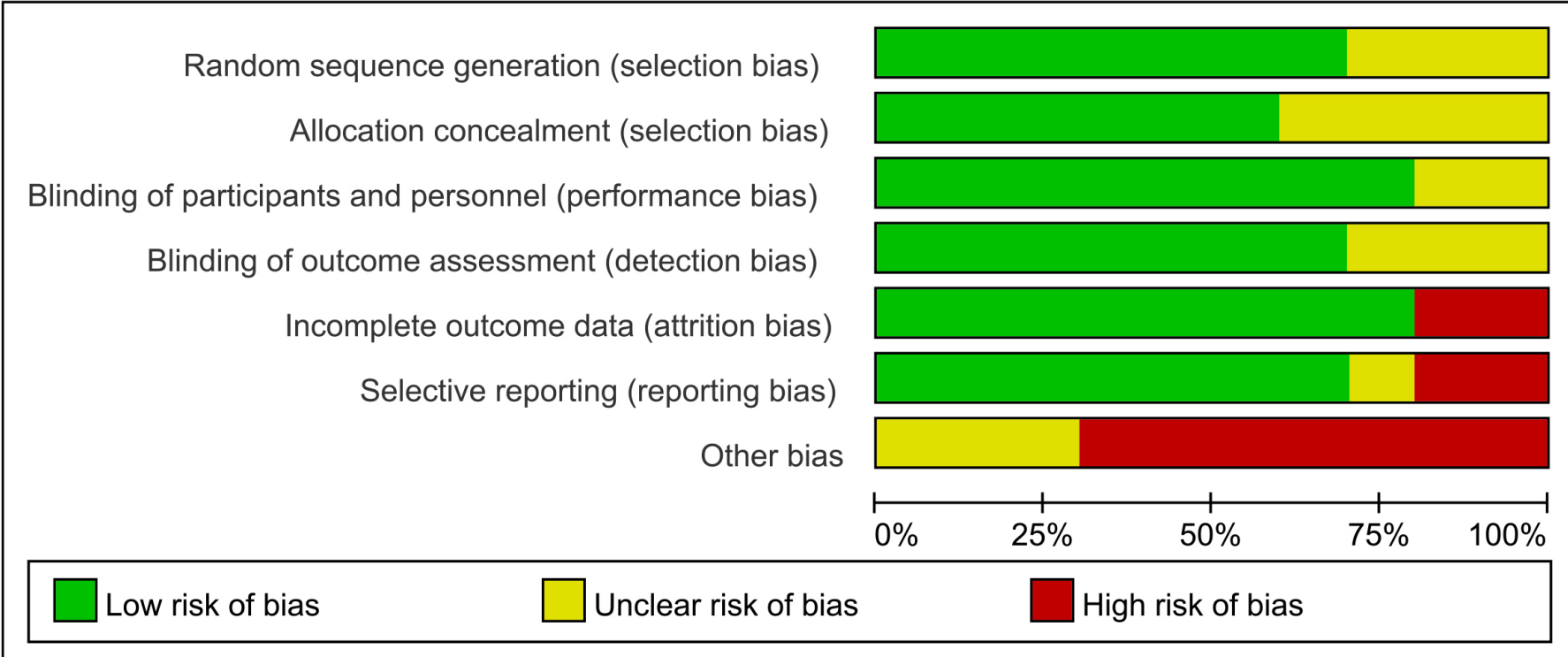

Figure 2 Risk of bias summary.

had significantly lower pain scores compared with the placebo during the late postoperative period (WMD $-2.10,95 \%$ CI -3.30 to $-0.90, \mathrm{p}<0.001$; one study, 20 participants). There is a paucity of data regarding acute pain relief efficacy of ketamine in this route during the early postoperative period.

\section{Cumulative morphine consumption}

Six studies ${ }^{111214151825}$ were eligible for the assessment of cumulative morphine consumption during 0-24-hour and 0-48-hour postoperative periods (figure 7). Among them, the combined data of five studies ${ }^{11} 12141525$ showed that the cumulative morphine consumption in the intravenous administration of ketamine group was significantly lower than that in the control group both during the 0-24hour (WMD $-17.76,95 \%$ CI -31.25 to -4.27 , $\mathrm{p}=0.01$; five studies, 366 participants) and 0-48-hour (WMD -21.79, $95 \%$ CI -25.46 to $-18.11, \mathrm{p}<0.001$; four studies, 252 participants) postoperative periods. Substantial heterogeneity was observed during the 0-24-hour postoperative period $\left(\mathrm{I}^{2}=95 \%\right.$; $\left.\mathrm{p}<0.001\right)$, but not during the 0-48-hour postoperative period $\left(\mathrm{I}^{2}=0 \% ; \mathrm{p}=0.76\right)$. Funnel plot did not reveal any substantial asymmetry (figure 6), and Begg's rank correlation test did not indicate publication bias among the included studies (0-24-hour: $p=0.806$; 0-48hour: $\mathrm{p}=1.000$ ).

In addition, only one study ${ }^{18}$ reported that the intra-articular administration of ketamine significantly decreased the cumulative morphine consumption compared with placebo during the 0-48-hour postoperative period (WMD $-4.10,95 \%$ CI -5.85 to -2.35 , p<0.001; one study, 44 participants), but not during the 0-24-hour postoperative period (WMD $-0.40,95 \% \mathrm{CI}-1.83$ to $1.03, \mathrm{p}=0.58$; one study, 44 participants). No study was conducted focusing on whether epidural ketamine was effective in reducing cumulative morphine consumption.

\section{Adverse effects}

In the present study, psychotic adverse effects include psychomimetic effect, drowsiness, nightmare, hallucination and delirium, while gastrointestinal adverse effects include nausea and vomiting. A total of seven studies $^{1112} 14$ 16-18 24 were eligible for the assessment of psychotic and gastrointestinal adverse effects (figures 8 and 9). The analysis of pooled data revealed that the incidence of adverse effects in the intravenous administration of ketamine group was significantly lower than that in the control group with regard to gastrointestinal adverse effects (RR 0.67, 95\% CI 0.51 to $0.89, \mathrm{p}=0.005$; four studies, 335 participants), but not to psychotic adverse effects (RR 0.94; 95\% CI 0.59 to $1.50 ; \mathrm{p}=0.79$; three studies, 286 participants). No evidence of heterogeneity was observed (gastrointestinal: $\mathrm{I}^{2}=32 \%, \mathrm{p}=0.22$; psychotic: $\left.\mathrm{I}^{2}=0 \%, \mathrm{p}=0.98\right)$. Funnel plot did not reveal any substantial asymmetry (figure 6), and Begg's rank correlation test did not indicate publication bias among the included studies (gastrointestinal: $\mathrm{p}=1.000$; psychotic: $\mathrm{p}=1.000$ ).

The combined data of two studies ${ }^{1718}$ showed that there were no significant differences between the intra-articular administration of ketamine group and control group with regard to gastrointestinal adverse effects (RR 1.00, $95 \%$ CI 0.53 to $1.89, \mathrm{p}=0.99$; two studies, 79 participants), with no substantial heterogeneity $\left(\mathrm{I}^{2}=0 \% ; \mathrm{p}=0.51\right)$. Funnel plot did not reveal any substantial asymmetry (figure 6), and Begg's rank correlation test did not indicate publication bias among the included studies $(p=1.000)$. In addition, one study ${ }^{17}$ reported that there was no significant difference between the intra-articular administration of ketamine group and the control group with regard to psychotic adverse effects (RR 1.05, 95\% CI 0.36 to 3.07, $\mathrm{p}=0.93$; one study, 39 participants).

Only one study, ${ }^{24}$ conducted on a small-sized sample, reported that the incidence of adverse effects in the 


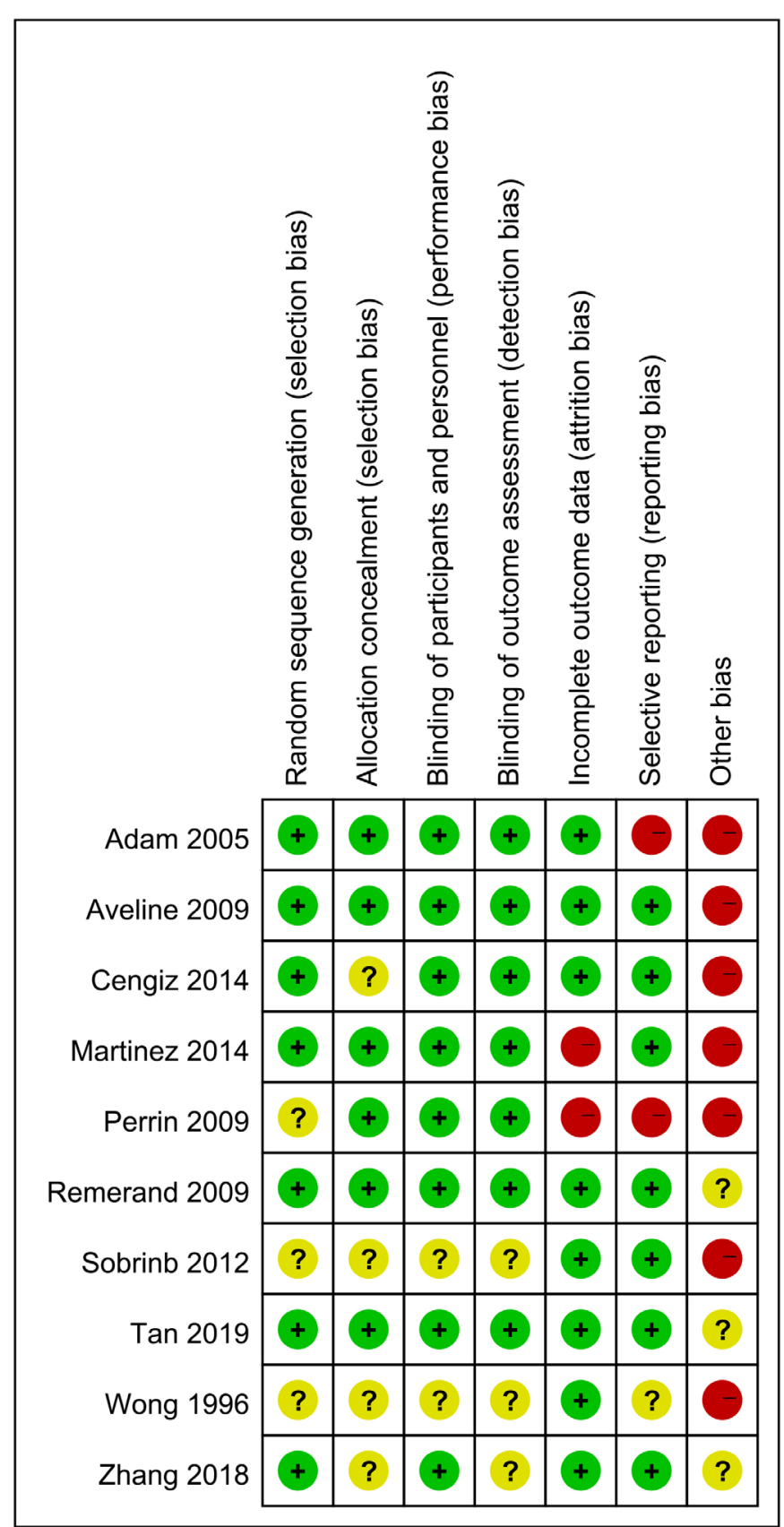

Figure 3 Risk of bias graph.

epidural administration of ketamine group was significantly lower than that in the control group with regard to gastrointestinal adverse effects (RR $0.06,95 \%$ CI 0.00 to $0.90, \mathrm{p}=0.04$; one study, 20 participants), but not to psychotic adverse effects (RR $0.67,95 \%$ CI 0.27 to 1.66 , $\mathrm{p}=0.38$; one study, 20 participants).

\section{DISCUSSION}

Based on newly accumulated evidence, the present study showed that the intravenous administration of ketamine in patients undergoing total knee or hip arthroplasty was effective for pain relief and the reduction of cumulative morphine consumption without increasing the risk of gastrointestinal or psychotic adverse effects. In addition, the limited data available for intra-articular and epidural administration of ketamine pointed to a significant reduction in pain intensity during the late postoperative period. However, due to insufficiency of evidence, findings with regard to these two administration routes need to be confirmed by more quantitative studies.

The efficacy and safety of the administration of ketamine for postoperative pain relief has been examined for decades, but the findings are still contradictory and inconclusive. ${ }^{9}{ }^{26-28}$ A previous Cochrane review published in 2006, which included 37 trials involving different routes of administration (ie, intravenous, intramuscular and epidural),${ }^{10}$ suggested that ketamine in subanaesthetic dose was effective in reducing the demand of morphine during the first 24 hours after surgery with no major complications. The most recent Cochrane review up to date including 130 trials using the intravenous administration of ketamine demonstrated that perioperative intravenous ketamine could probably reduce postoperative analgesic consumption and pain intensity. ${ }^{9}$ In addition, some reviews also focused on the efficacy and safety of ketamine for postoperative pain relief with respect to different types of surgery, ${ }^{26-28}$ but no systematic review or meta-analysis confined their participants to total knee or hip arthroplasty. Moreover, the deviation of the overall results may be largely attributed to the lack of a uniform administration route of ketamine. In the present study, a total of 10 studies targeting at patients undergoing total knee or hip arthroplasty were eligible for the assessment of acute postoperative pain intensity, and the analyses were also stratified by different administration routes of ketamine.

Morphine, a mu-opioid receptor agonist, relieves pain by binding and activating the receptors in both the central and peripheral nervous systems. ${ }^{29}$ As the most widely used narcotic for postoperative pain control, it has been reported of a high incidence of adverse effects including nausea and vomiting, which may severely impede postoperative convalescence. ${ }^{3031}$ On the other hand, ketamine, an NMDA receptor antagonist, was deemed to inhibit the sensitisation of nociceptive pathways and prevent the activation of the pronociceptive system associated with opiates and opiate tolerance. ${ }^{32-37}$ Cengiz et al ${ }^{11}$ reported that the intraoperative continuous low-dose infusion of ketamine could reduce postoperative pain and cumulative morphine consumption without increasing the incidence of adverse effects in patients undergoing total knee arthroplasty. Aveline et $a l^{12}$ demonstrated that ketamine could produce opioid-sparing, so as to alleviate pain intensity and improve mobilisation after total knee arthroplasty. Comparatively, the effect of nefopam was less significant in the same circumstances. Wong et $a l^{24}$ suggested that although ketamine did not exert a significant pain relief effect directly for patients undergoing total knee or hip arthroplasty, it could enhance the analgesic effect of morphine, lower the dosage of morphine demanded and mitigate the various adverse effects. In the present study, the combined data showed that the 


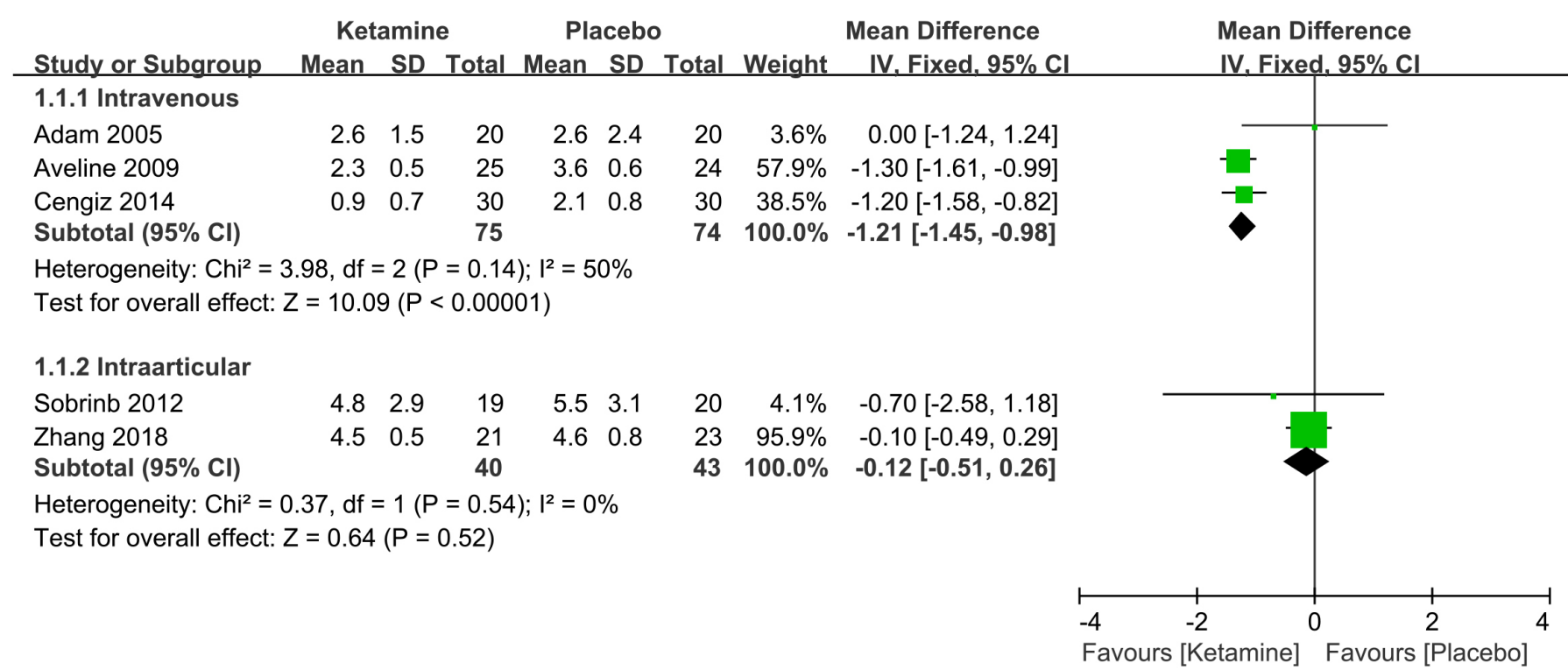

Figure 4 Forest plot of pain intensity in the early (0-8-hour) postoperative period.

cumulative morphine consumption in the intravenous administration of ketamine group was significantly lower than that in the control group during both 0-24-hour and 0-48-hour postoperative periods, and the intra-articular administration of ketamine could also reduce the cumulative morphine consumption during the 0-48-hour postoperative period. This is the first study that investigates the morphine-sparing effect of different administration routes of ketamine after knee or hip arthroplasty through meta-analysis.

It has been well established that opioid is generally effective for pain relief, but often incurs side-effects (eg, nausea, vomiting and hallucinations) that can prolong the length of hospitalisation. ${ }^{1}$ Therefore, the clinical value of the morphine-sparing effect of ketamine may be weakened if opioid-related side effects cannot be mitigated. ${ }^{38}$ The pooled data of this meta-analysis showed that psychotic and gastrointestinal adverse effects of the intravenous and intra-articular administration of ketamine in total knee or hip arthroplasty patients were similar to, or even less that of saline controls. This suggests that both the intravenous and intra-articular administration of ketamine can be considered as a recommendation for patients scheduled for total knee or hip arthroplasty.

The occurrence of central sensitisation, which may amplify postoperative pain, has been deemed a risk factor of chronic pain after surgery. ${ }^{39}$ A 12-month follow-up study of a previous randomised controlled trial (a total of

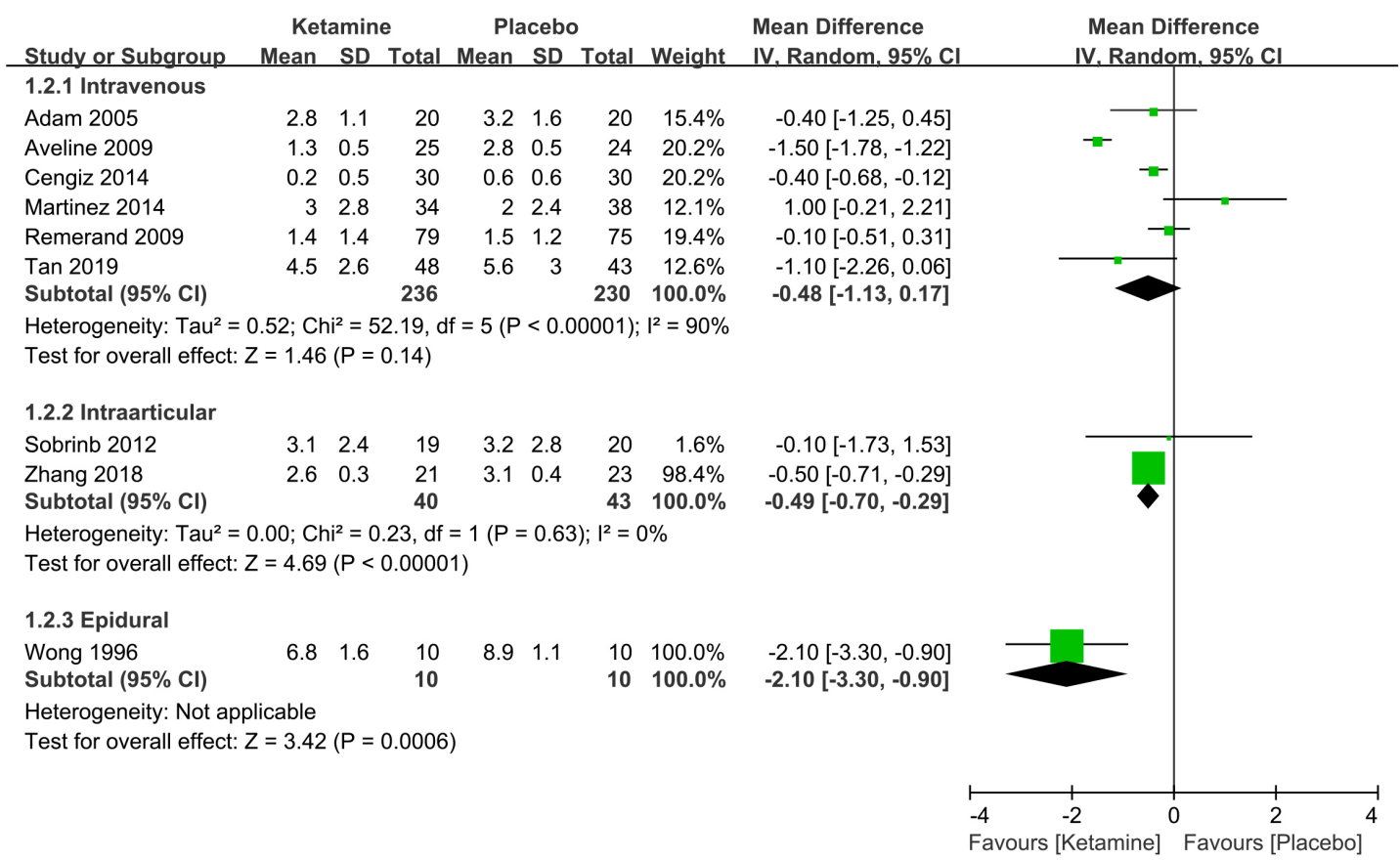

Figure 5 Forest plot of pain intensity in the late (8-24-hour) postoperative period. 


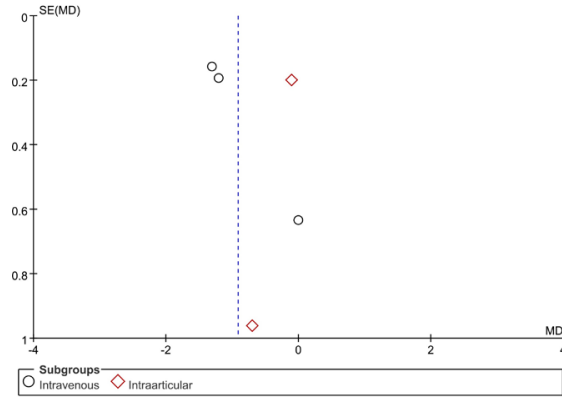

(A) VAS of post-operative pain intensity in the early period
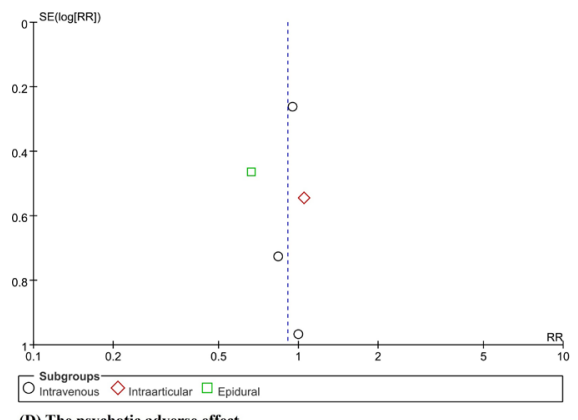

(D) The psychotic adverse effect

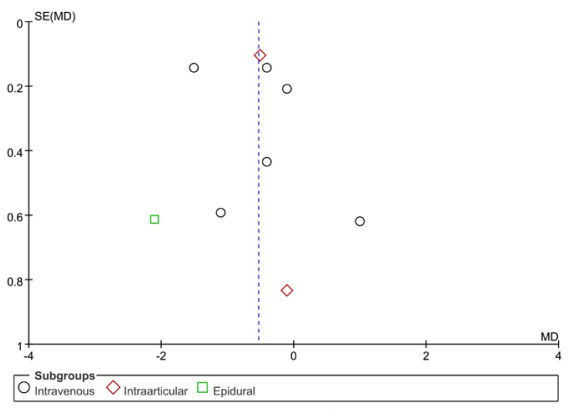

(B) VAS of post-operative pain intensity in the late period

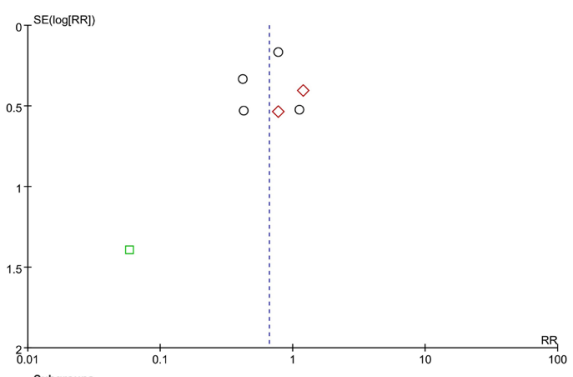

$\mathrm{O}_{\text {Intravenous }}^{\text {Subgrous }} \diamond_{\text {Intraarticular }} \square_{\text {Epidural }}$

(E) The gastrointestinal adverse effect

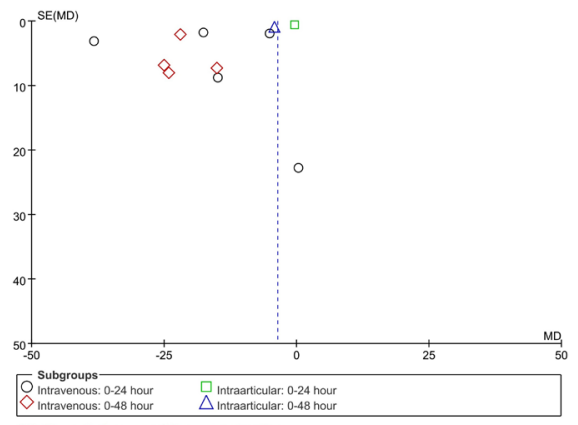

(C) Cumulative morphine consumption

Figure 6 Funnel plots.

69 patients completed the trial) was conducted to determine the prevalence and the risk factors of chronic pain after total knee arthroplasty and to assess the role of the perioperative intravenous administration of ketamine. ${ }^{40}$ Its results showed that the prevalence of chronic pain at 12 months was $12.5 \%$ in the ketamine group and $26.1 \%$ in the

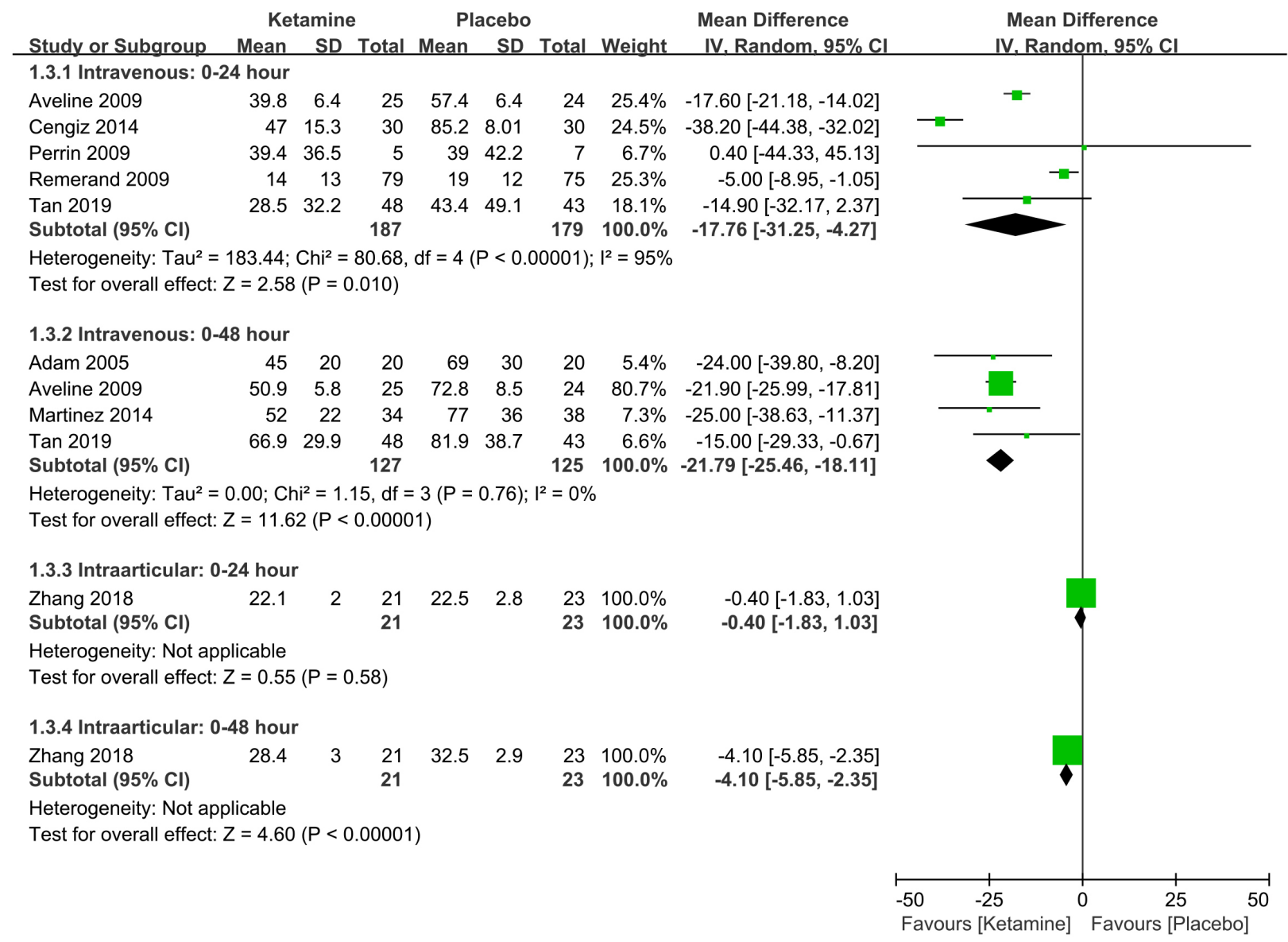

Figure 7 Forest plot of the cumulative morphine consumption. 


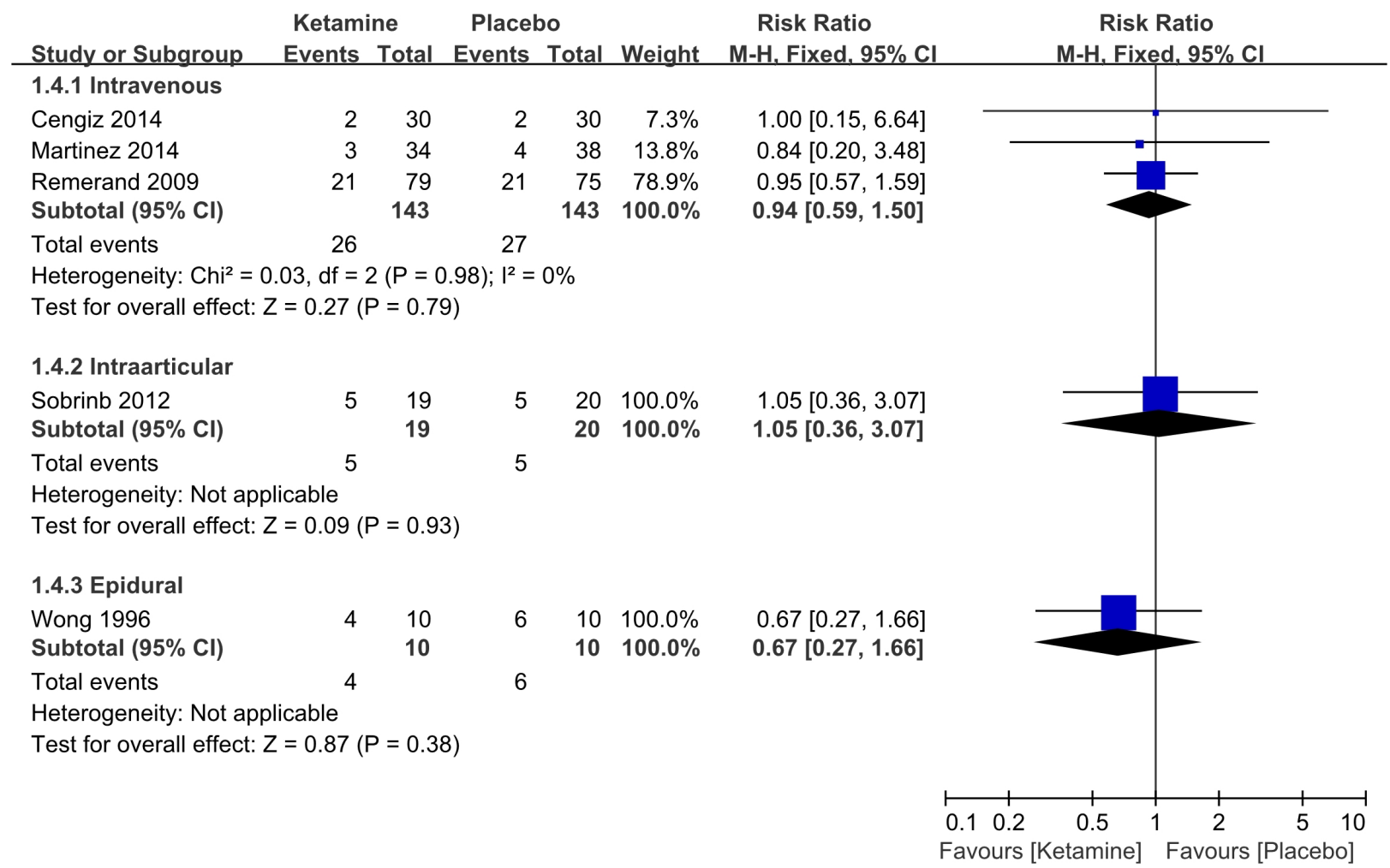

Figure 8 Forest plot of the psychotic adverse effects.

control group. Based on the results of long-term follow-up, ketamine can also reduce the incidence of neuropathic pain and improve knee flexion and physical functions compared with the controls. This implies that the intravenous administration of ketamine may improve the long-term outcome of rehabilitation for patients after total knee arthroplasty.

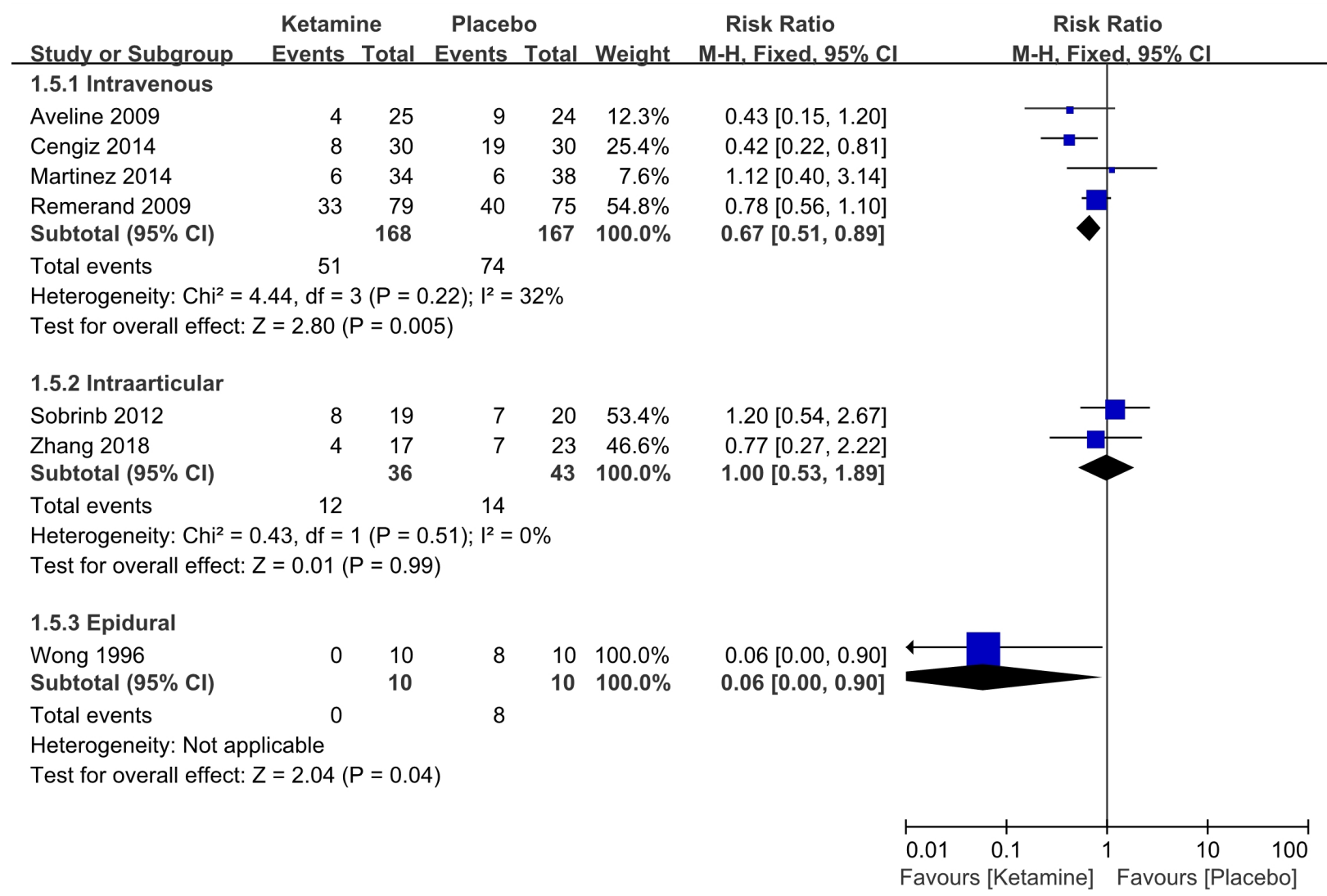

Figure 9 Forest plot of the gastrointestinal adverse effects. 
Intra-articular injection is simple and requires no special expertise or equipment. Ketamine is rarely used intra-articularly, but research findings suggest that this drug may be used as an adjuvant analgesic administered by intra-articular injection after knee arthroscopy. ${ }^{41}{ }^{42}$ Lawland et $a t^{43}$ concluded that, because the peripheral administration of NMDA and non-NMDA receptor antagonists directly into the joint was shown to have an antihyperalgesic effect, both NMDA and non-NMDA receptors were activated in the joint. Furthermore, in addition to chondrocytes, ${ }^{44}$ NMDA receptor was also evidence to be expressed in human synoviocytes. ${ }^{45} 46$ Thus, it may explain, at least in part, why the intra-articular administration of ketamine is probably effective for postoperative analgesia in patients undergoing total knee or hip arthroplasty.

This is the first meta-analysis that directly investigates the efficacy and safety of ketamine in all feasible administration routes after total knee or hip arthroplasty. However, several limitations of this study should be acknowledged. First, substantial heterogeneity was observed in part of the results. Although some studies had been eliminated from sensitivity analyses based on appropriate exclusion criteria, the source of heterogeneity was not identified. Second, the lack of uniform doses of ketamine and start time of injection may contribute to the deviation of the overall results in the present study. Third, the meta-analysis technique is not fully applicable in investigating the efficacy and safety of the intra-articular and epidural administration of ketamine due to the small number of included studies. Therefore, the statistical power of the related sections was affected to a certain extent.

\section{CONCLUSIONS}

Intravenous administration of ketamine is effective and safe for postoperative pain relief in patients undergoing total knee or hip arthroplasty. Nevertheless, the analgesic efficacy and safety of ketamine in such patients seem to vary by different administration routes and still warrants further studies to explore.

Contributors All authors had full access to the data in the study and take responsibility for the integrity of the data and the accuracy of the data analysis. $\mathrm{DX}$ and $\mathrm{XD}$ conceived the study. BX and YW were responsible for conception and design of the study and drafted the manuscript. $\mathrm{CZ}, \mathrm{HH}, \mathrm{ZW}$ and $\mathrm{JL}$ contributed to data collection. JW and GL contributed to preparation and data analysis. BX and YW contributed to study retrieval. GL and DX contributed to revision of the manuscript. All the authors contributed to the interpretation of the data and critically reviewed the manuscript for publication.

Funding This work was supported by the Innovation Foundation of the Central South University for Postgraduate (2018zzts045 and 2018zzts256), the National Natural Science Foundation of China $(81772413,81472130,81672225,81601941$, 81501923, 81702207 and 81702206), the Scientific Research Project of Science and Technology Office of Hunan Province (2017TP1005), the Key Research and Development Program of Hunan Province (2018SK2070), the Young Investigator Grant of Xiangya Hospital, Central South University (2016Q03 and 2016Q06), the Xiangya Clinical Big Data System Construction Project of Central South University (45), the Clinical Scientific Research Foundation of Xiangya Hospital, Central South University (2015L03), the Postdoctoral Science Foundation of Central South University (182130) and the Natural Science Foundation of Hunan Province (2017JJ3491, 2017JJ3492 and 2018JJ3825).
Competing interests None declared.

\section{Patient consent for publication Not required.}

Provenance and peer review Not commissioned; externally peer reviewed.

Data availability statement Data are available upon reasonable request.

Open access This is an open access article distributed in accordance with the Creative Commons Attribution Non Commercial (CC BY-NC 4.0) license, which permits others to distribute, remix, adapt, build upon this work non-commercially, and license their derivative works on different terms, provided the original work is properly cited, appropriate credit is given, any changes made indicated, and the use is non-commercial. See: http://creativecommons.org/licenses/by-nc/4.0/.

\section{REFERENCES}

1. Atchabahian A, Schwartz G, Hall CB, et al. Regional analgesia for improvement of long-term functional outcome after elective large joint replacement. Cochrane Database Syst Rev 2015;(8).

2. Farr J, Jaggers $\mathrm{R}$, Lewis $\mathrm{H}$, et al. Evidence-Based approach of treatment options for postoperative knee pain. Phys Sportsmed 2014;42:58-70.

3. Anastase DM, Cionac Florescu S, Munteanu AM, et al. Analgesic techniques in hip and knee arthroplasty: from the daily practice to evidence-based medicine. Anesthesiol Res Pract 2014;2014:1-8.

4. Souzdalnitski D, Halaszynski TM, Faclier G. Regional anesthesia and co-existing chronic pain. Curr Opin Anaesthesiol 2010;23:662-70.

5. Angst MS, Clark JD. Ketamine for managing perioperative pain in opioid-dependent patients with chronic pain. Anesthesiology 2010;113:1-5.

6. Ketamine GJ. Cl-581) in clinical anaesthesia. Acta Anaesthesiol Scand 1968;12:15-21.

7. McCartney CJL, Sinha A, Katz J. A qualitative systematic review of the role of $\mathrm{N}$-methyl-D-aspartate receptor antagonists in preventive analgesia. Anesth Analg 2004;98:1385-400.

8. Elia N, Tramèr MR. Ketamine and postoperative pain - a quantitative systematic review of randomised trials. Pain 2005;113:61-70.

9. Brinck EC, Tiippana E, Heesen M, et al. Perioperative intravenous ketamine for acute postoperative pain in adults. Cochrane Database Syst Rev 2018;12.

10. Bell RF, Dahl JB, Moore RA, et al. Perioperative ketamine for acute postoperative pain. Cochrane Database Syst Rev 2006.

11. Cengiz P, Gokcinar D, Karabeyoglu I, et al. Intraoperative low-dose ketamine infusion reduces acute postoperative pain following total knee replacement surgery: a prospective, randomized double-blind placebo-controlled trial. $J$ Coll Physicians Surg Pak 2014;24:299-303.

12. Aveline C, Gautier JF, Vautier P, et al. Postoperative analgesia and early rehabilitation after total knee replacement: a comparison of continuous low-dose intravenous ketamine versus nefopam. Eur J Pain 2009;13:613-9.

13. Adam F, Chauvin M, Du Manoir B, et al. Small-dose ketamine infusion improves postoperative analgesia and rehabilitation after total knee arthroplasty. Anesthesia \& Analgesia 2005;100:475-80.

14. Remérand F, Le Tendre C, Baud A, et al. The early and delayed analgesic effects of ketamine after total hip arthroplasty: a prospective, randomized, controlled, double-blind study. Anesth Analg 2009;109:1963-71.

15. Tan TL, Longenecker AS, Rhee JH, et al. Intraoperative ketamine in total knee arthroplasty does not decrease pain and narcotic consumption: a prospective randomized controlled trial. $J$ Arthroplasty 2019.

16. Martinez V, Cymerman A, Ben Ammar S, et al. The analgesic efficiency of combined pregabalin and ketamine for total hip arthroplasty: a randomised, double-blind, controlled study. Anaesthesia 2014;69:46-52.

17. Guará Sobrinho H, Garcia JBS, Vasconcelos JW, et al. Analgesic efficacy of the intra-articular administration of $S(+)$ - ketamine in patients undergoing total knee arthroplasty. Brazilian Journal of Anesthesiology 2012;62:665-75.

18. Zhang J, Shi K, Jia H. Ketamine and bupivacaine attenuate post-operative pain following total knee arthroplasty: a randomized clinical trial. Exp Ther Med 2018;15.

19. Liberati A, Altman DG, Tetzlaff J, et al. The PRISMA statement for reporting systematic reviews and meta-analyses of studies that evaluate health care interventions: explanation and elaboration. $J$ Clin Epidemiol 2009;62:e1-34.

20. Hozo SP, Djulbegovic B, Hozo I. Estimating the mean and variance from the median, range, and the size of a sample. BMC Med Res Methodol 2005;5:13. 
21. Higgins J, Green S. Cochrane Handbook for Systematic Reviews of Interventions Version 5.1.0 [updated March 2011]: The Cochrane Collaboration 2011.

22. Zeng $\mathrm{C}$, Wei J, Li H, et al. Effectiveness and safety of glucosamine, chondroitin, the two in combination, or celecoxib in the treatment of osteoarthritis of the knee. Sci Rep 2015;5:16827.

23. Begg CB, Mazumdar M. Operating characteristics of a RANK correlation test for publication bias. Biometrics 1994;50:1088-101.

24. Wong CS, Liaw WJ, Tung CS, et al. Ketamine potentiates analgesic effect of morphine in postoperative epidural pain control. Reg Anesth 1996;21:534-41.

25. Perrin SB, Purcell AN. Intraoperative ketamine may influence persistent pain following knee arthroplasty under combined general and spinal anaesthesia: a pilot study. Anaesth Intensive Care 2009;37:248-53.

26. Ye F, Wu Y, Zhou C. Effect of intravenous ketamine for postoperative analgesia in patients undergoing laparoscopic cholecystectomy. Medicine 2017;96:e9147.

27. Moyse DW, Kaye AD, Diaz JH, et al. Perioperative ketamine administration for thoracotomy pain. Pain Physician 2017:20:173-84.

28. Pendi A, Field R, Farhan SD, et al. Perioperative ketamine for analgesia in spine surgery: a meta-analysis of randomized controlled trials. Spine 2018;43:E299-307.

29. Shimizu N, Kishioka S, Maeda T, et al. Involvement of peripheral mechanism in the verapamil-induced potentiation of morphine analgesia in mice. J Pharmacol Sci 2004;95:452-7.

30. Bromage PR, Camporesi E, Chestnut D. Epidural narcotics for postoperative analgesia. Anesth Analg 1980:473-80.

31. Bromage PR, Camporesi EM, Durant PAC, et al. Nonrespiratory side effects of epidural morphine. Anesthesia \& Analgesia 1982;61:490???495-5.

32. Chizh BA. Low dose ketamine: a therapeutic and research tool to explore N-methyl-D-aspartate (NMDA) receptor-mediated plasticity in pain pathways. J Psychopharmacol 2007;21:259-71.

33. Kissin I, Bright CA, Bradley EL. The effect of ketamine on opioidinduced acute tolerance: can it explain reduction of opioid consumption with ketamine-opioid analgesic combinations? Anesthesia \& Analgesia 2000;91:1483-8.

34. Willert RP, Woolf CJ, Hobson AR, et al. The development and maintenance of human visceral pain hypersensitivity is dependent on the N-methyl-D-aspartate receptor. Gastroenterology 2004;126:683-92.

35. Celerier E, Rivat C, Jun $\mathrm{Y}$, et al. Long-Lasting hyperalgesia induced by fentanyl in rats: preventive effect of ketamine. Anesthesiology 2000;92:465-72.

36. Lee SK. The use of ketamine for perioperative pain management. Korean J Anesthesiol 2012;63:1.

37. Minville V, Fourcade O, Girolami J-P, et al. Opioid-Induced hyperalgesia in a mice model of orthopaedic pain: preventive effect of ketamine †. Br J Anaesth 2010;104:231-8.

38. Kehlet H. Postoperative opioid sparing to hasten recovery: what are the issues? Anesthesiology 2005;102:1083-5.

39. Latremoliere A, Woolf CJ. Central sensitization: a generator of pain hypersensitivity by central neural plasticity. The Journal of Pain 2009;10:895-926.

40. Aveline $\mathrm{C}$, Roux AL, Hetet HL, et al. Pain and recovery after total knee arthroplasty: a 12-month follow-up after a prospective randomized study evaluating nefopam and ketamine for early rehabilitation. Clin J Pain 2014;30:749-54.

41. Menigaux C, Guignard B, Fletcher D, et al. Intraoperative small-dose ketamine enhances analgesia after outpatient knee arthroscopy. Anesth Analg 2001;93:606-12.

42. Dal D, Tetik O, Altunkaya $\mathrm{H}$, et al. The efficacy of intra-articular ketamine for postoperative analgesia in outpatient arthroscopic surgery. Arthroscopy: The Journal of Arthroscopic \& Related Surgery 2004;20:300-5.

43. Lawand NB, Willis WD, Westlund KN. Excitatory amino acid receptor involvement in peripheral nociceptive transmission in rats. Eur $\mathrm{J}$ Pharmacol 1997;324:169-77.

44. Ramage L, Martel M-A, Hardingham GE, et al. Nmda receptor expression and activity in osteoarthritic human articular chondrocytes. Osteoarthritis Cartilage 2008;16:1576-84.

45. McNearney TA, Ma Y, Chen Y, et al. A peripheral neuroimmune link: glutamate agonists upregulate NMDA NR1 receptor mRNA and protein, vimentin, TNF- $\alpha$, and RANTES in cultured human synoviocytes. Am J Physiol Regul Integr Comp Physiol 2010;298:R58 4-R598.

46. Flood S, Parri R, Williams A, et al. Modulation of interleukin-6 and matrix metalloproteinase 2 expression in human fibroblast-like synoviocytes by functional ionotropic glutamate receptors. Arthritis Rheum 2007;56:2523-34. 